\title{
Cis influence and the Co-C Bond Reactivity Relationship in Benzylcobaloximes with Glyoxime and Dimesitylglyoxime
}

Mouchumi Bhuyan, Moitree Laskar, Debaprasad Mandal and B. D. Gupta*

Department of Chemistry, Indian Institute of Technology, Kanpur, 208 016, India bdg@iitk.ac.in Tel: +91-512-2597046; Fax: +91-512-2597436

\section{Supporting Information}

Table S1. Yield and Elemental Analysis Data for 1-10 and 1a-10a

\begin{tabular}{|l|l|l|l|l|}
\hline & & \multicolumn{3}{|c|}{ \% found (calcd.) } \\
\hline no. & formula & \multicolumn{1}{|c|}{$\mathrm{C}$} & \multicolumn{1}{c|}{$\mathrm{H}$} & \multicolumn{1}{|c|}{$\mathrm{N}$} \\
\hline 1 & $\mathrm{C}_{52} \mathrm{H}_{58} \mathrm{Co}_{1} \mathrm{~N}_{5} \mathrm{O}_{4}$ & $71.02(71.30)$ & $6.64(6.67)$ & $7.96(7.99)$ \\
\hline 2 & $\mathrm{C}_{52} \mathrm{H}_{57} \mathrm{ClCo}_{1} \mathrm{~N}_{5} \mathrm{O}_{4}$ & $68.35(68.60)$ & $6.33(6.31)$ & $7.72(7.69)$ \\
\hline 3 & $\mathrm{C}_{53} \mathrm{H}_{57} \mathrm{Co}_{1} \mathrm{~N}_{6} \mathrm{O}_{4}$ & $70.42(70.68)$ & $6.36(6.38)$ & $9.36(9.33)$ \\
\hline 4 & $\mathrm{C}_{53} \mathrm{H}_{60} \mathrm{Co}_{1} \mathrm{~N}_{5} \mathrm{O}_{5}$ & $70.51(70.26)$ & $6.65(6.68)$ & $7.70(7.73)$ \\
\hline 5 & $\mathrm{C}_{56} \mathrm{H}_{60} \mathrm{Co}_{1} \mathrm{~N}_{5} \mathrm{O}_{4}$ & $72.39(72.63)$ & $6.55(6.53)$ & $7.59(7.56)$ \\
\hline 6 & $\mathrm{C}_{16} \mathrm{H}_{18} \mathrm{Co}_{1} \mathrm{~N}_{5} \mathrm{O}_{4}$ & $47.44(47.65)$ & $4.48(4.50)$ & $17.35(17.37)$ \\
\hline 7 & $\mathrm{C}_{16} \mathrm{H}_{17} \mathrm{ClCo}_{1} \mathrm{~N}_{5} \mathrm{O}_{4}$ & $43.73(43.90)$ & $3.92(3.91)$ & $16.05(16.00)$ \\
\hline 8 & $\mathrm{C}_{17} \mathrm{H}_{17} \mathrm{Co}_{1} \mathrm{~N}_{6} \mathrm{O}_{4}$ & $47.85(47.67)$ & $3.99(4.00)$ & $19.55(19.62)$ \\
\hline 9 & $\mathrm{C}_{17} \mathrm{H}_{20} \mathrm{Co}_{1} \mathrm{~N}_{5} \mathrm{O}_{5}$ & $47.31(47.12)$ & $4.63(4.65)$ & $16.22(16.16)$ \\
\hline 10 & $\mathrm{C}_{20} \mathrm{H}_{20} \mathrm{Co}_{1} \mathrm{~N}_{5} \mathrm{O}_{4}$ & $53.19(52.99)$ & $4.47(4.45)$ & $15.37(15.45)$ \\
\hline $1 \mathrm{a}$ & $\mathrm{C}_{52} \mathrm{H}_{58} \mathrm{Co}_{1} \mathrm{~N}_{5} \mathrm{O}_{6}$ & $68.52(68.79)$ & $6.46(6.44)$ & $7.68(7.71)$ \\
\hline $2 \mathrm{a}$ & $\mathrm{C}_{52} \mathrm{H}_{57} \mathrm{ClCo}_{1} \mathrm{~N}_{5} \mathrm{O}_{6}$ & $66.01(66.27)$ & $6.08(6.10)$ & $7.42(7.43)$ \\
\hline $3 \mathrm{a}$ & $\mathrm{C}_{53} \mathrm{H}_{57} \mathrm{Co}_{1} \mathrm{~N}_{6} \mathrm{O}_{6}$ & $68.47(68.23)$ & $6.14(6.16)$ & $8.98(9.01)$ \\
\hline $4 \mathrm{a}$ & $\mathrm{C}_{53} \mathrm{H}_{60} \mathrm{Co}_{1} \mathrm{~N}_{5} \mathrm{O}_{7}$ & $67.65(67.86)$ & $6.43(6.45)$ & $7.44(7.47)$ \\
\hline $5 \mathrm{a}$ & $\mathrm{C}_{56} \mathrm{H}_{60} \mathrm{Co}_{1} \mathrm{~N}_{5} \mathrm{O}_{6}$ & $69.97(70.21)$ & $6.29(6.31)$ & $7.33(7.31)$ \\
\hline $6 \mathrm{a}$ & $\mathrm{C}_{16} \mathrm{H}_{18} \mathrm{Co}_{1} \mathrm{~N}_{5} \mathrm{O}_{6}$ & $44.34(44.15)$ & $4.19(4.17)$ & $16.04(16.09)$ \\
\hline $7 \mathrm{a}$ & $\mathrm{C}_{16} \mathrm{H}_{17} \mathrm{ClCo}_{1} \mathrm{~N}_{5} \mathrm{O}_{6}$ & $40.76(40.91)$ & $3.64(3.65)$ & $14.86(14.91)$ \\
\hline $8 \mathrm{a}$ & $\mathrm{C}_{17} \mathrm{H}_{17} \mathrm{Co}_{1} \mathrm{~N}_{6} \mathrm{O}_{6}$ & $44.18(44.36)$ & $3.73(3.72)$ & $18.32(18.26)$ \\
\hline $9 \mathrm{a}$ & $\mathrm{C}_{17} \mathrm{H}_{20} \mathrm{Co}_{1} \mathrm{~N}_{5} \mathrm{O}_{7}$ & $43.71(43.88)$ & $4.35(4.33)$ & $15.10(15.05)$ \\
\hline $10 \mathrm{a}$ & $\mathrm{C}_{20} \mathrm{H}_{20} \mathrm{Co}_{1} \mathrm{~N}_{5} \mathrm{O}_{6}$ & $49.32(49.49)$ & $4.17(4.15)$ & $14.38(14.43)$ \\
\hline & & & & \\
\hline
\end{tabular}




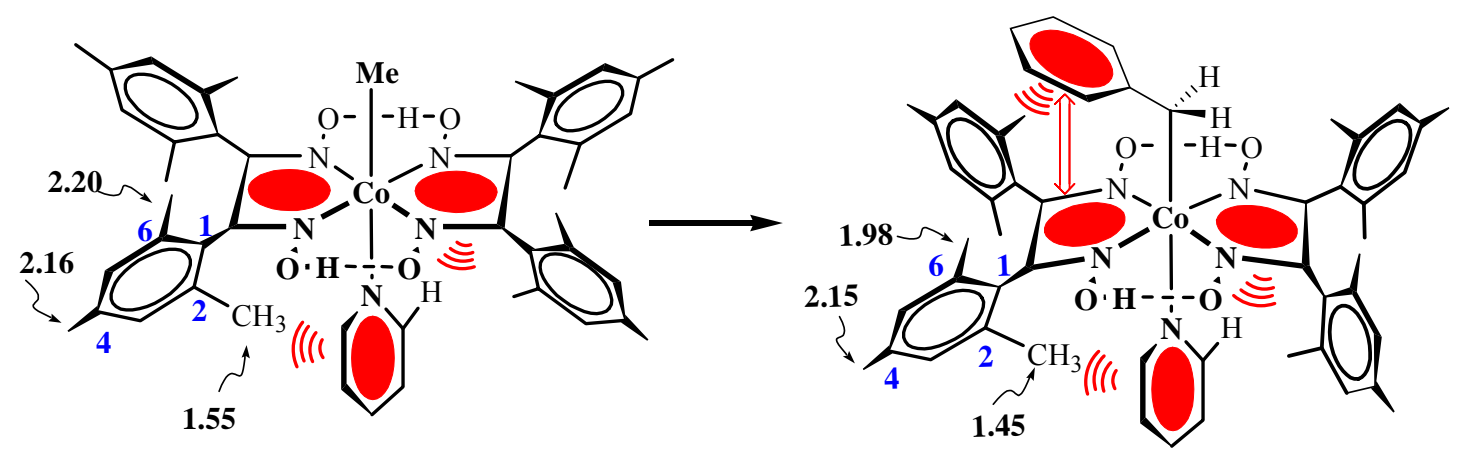

Figure S1. $\mathrm{RCo}(\mathrm{dmestgH})_{2} \mathrm{Py}$ and $\mathrm{PhCH}_{2} \mathrm{Co}(\mathrm{dmestgH})_{2} \mathrm{Py}$
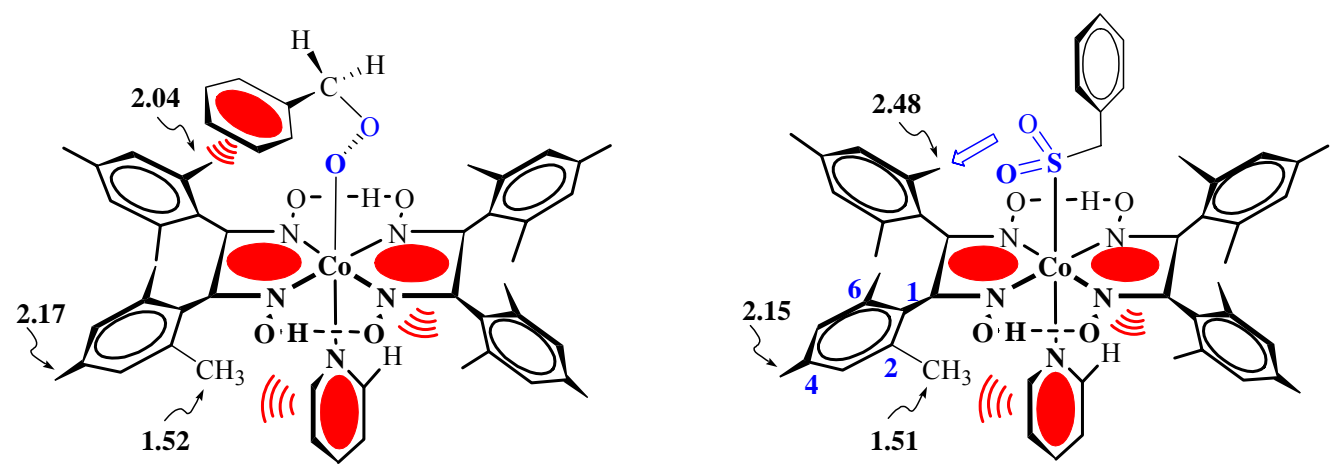

Figure S2. $\mathrm{PhCH}_{2}\left(\mathrm{O}_{2}\right) \mathrm{Co}(\mathrm{dmestgH})_{2} \mathrm{Py}$ and $\mathrm{PhCH}_{2}\left(\mathrm{SO}_{2}\right) \mathrm{Co}(\mathrm{dmestgH})_{2} \mathrm{Py}$

Table S2. $\Delta \delta\left({ }^{13} \mathrm{C}, \mathrm{C}=\mathrm{N}\right)\left(\delta_{\text {Complex }}-\delta_{\text {Free }}\right)$

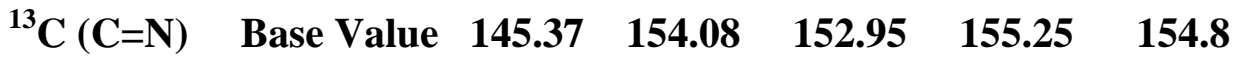

\begin{tabular}{|l|l|l|l|l|l|}
\hline $\mathrm{X}$ & $\mathrm{gH}$ & $\mathrm{dmgH}$ & chgH & dpgH & mestgH \\
\hline $\mathrm{Bn}$ & -7.37 & -4.75 & -2.79 & -4.22 & -1.53 \\
\hline 4-Cl & -7.27 & -4.72 & -2.79 & -4.12 & -1.38 \\
\hline 4-CN & -7.06 & -4.43 & -2.84 & -3.85 & -1.00 \\
\hline 3-OMe & -7.26 & -3.86 & -2.79 & -4.16 & -1.45 \\
\hline 2-Naph & & -4.69 & -2.73 & & -0.72 \\
\hline
\end{tabular}

\begin{tabular}{|l|l|l|l|l|l|}
\hline & & & & & \\
\hline Bn & 138.00 & 149.33 & 150.16 & 151.03 & 153.27 \\
\hline 4-Cl-Bn & 138.10 & 149.36 & 150.16 & 151.13 & 153.42 \\
\hline 4-CN-Bn & 138.31 & 149.65 & 150.11 & 151.40 & 153.80 \\
\hline 3-OMe-Bn & 138.11 & 150.22 & 150.16 & 151.09 & 153.35 \\
\hline 2-Naph & & 149.39 & 150.22 & & 154.08 \\
\hline
\end{tabular}




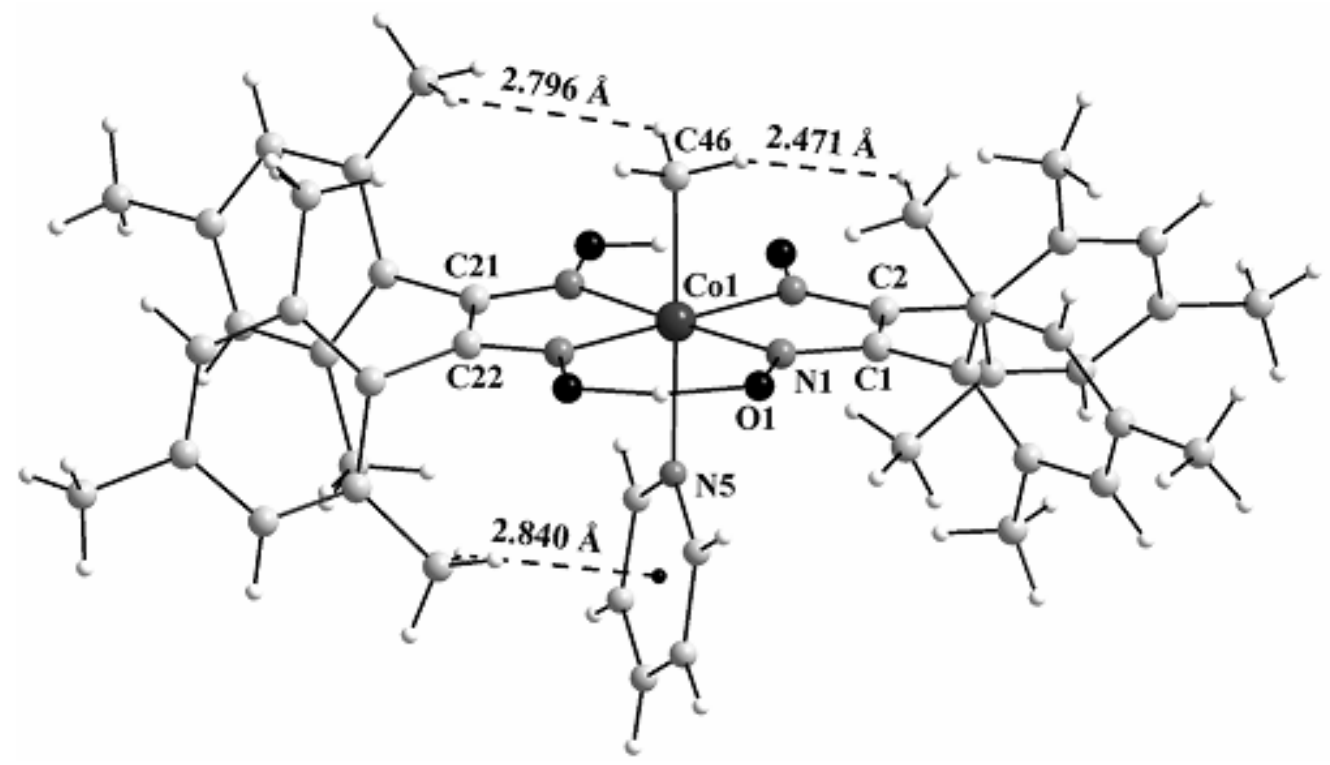

Figure S3. Steric crowding of axial positions in $\mathrm{MeCo}(\mathrm{dmestgH})_{2} \mathrm{Py}$ crystal structure.

Table S3. X-ray structure data comparison in $\mathrm{MeCo(dioximes)}{ }_{2} \mathrm{Py}$

\begin{tabular}{|l|c|c|c|c|}
\hline & $\mathrm{gH}$ & $\mathrm{dmgH}$ & $\mathrm{dpgH}$ & $\mathrm{dmestgH}$ \\
\hline $\mathrm{Co}-\mathrm{C}(\AA)$ & $2.005(4)$ & $1.998(5)$ & $1.997(4)$ & $2.002(3)$ \\
\hline $\mathrm{Co}-\mathrm{N}_{\mathrm{Py}}(\AA)$ & $2.064(3)$ & $2.086(4)$ & $2.053(4)$ & $2.085(3)$ \\
\hline$d(\AA)$ & +0.03 & +0.04 & +0.05 & -0.0177 \\
\hline$\alpha(\operatorname{deg})$ & 2.0 & 3.2 & 4.7 & 7.25 \\
\hline$\tau(\operatorname{deg})$ & 89.6 & 89.7 & 89.8 & 67.09 \\
\hline
\end{tabular}

Previously reported molecular structures, $(\mathrm{Me} / \mathrm{Cl} / \mathrm{Br}) \mathrm{Co}(\mathrm{dmestgH})_{2} \mathrm{Py},{ }^{8}$ are distinctive and the characteristic features (apart from $\mathrm{Co}-\mathrm{C}$ bond lengths, which defines the influence ${ }^{2,8}$ ) are: $d$ is always $-\mathrm{ve}, \alpha$ is very high and $\tau$ is always highly deviated from $90^{\circ}$, in contrast $d$ is always +ve, low $\alpha$ and $\tau \sim 90^{\circ}$ in other dioximes systems ( $\mathrm{gH}, \mathrm{dmgH}, \mathrm{dpgH}$ ). Benzyl analogue of dmestgH are even more strained as they are highly unstable in solution. It is interesting to note that after oxygen insertion the strain in the molecule gets released as $\alpha\left(3.17^{\circ}\right)$ is low and $d(+0.011 \AA)$ becomes similar to other cobaloximes. 


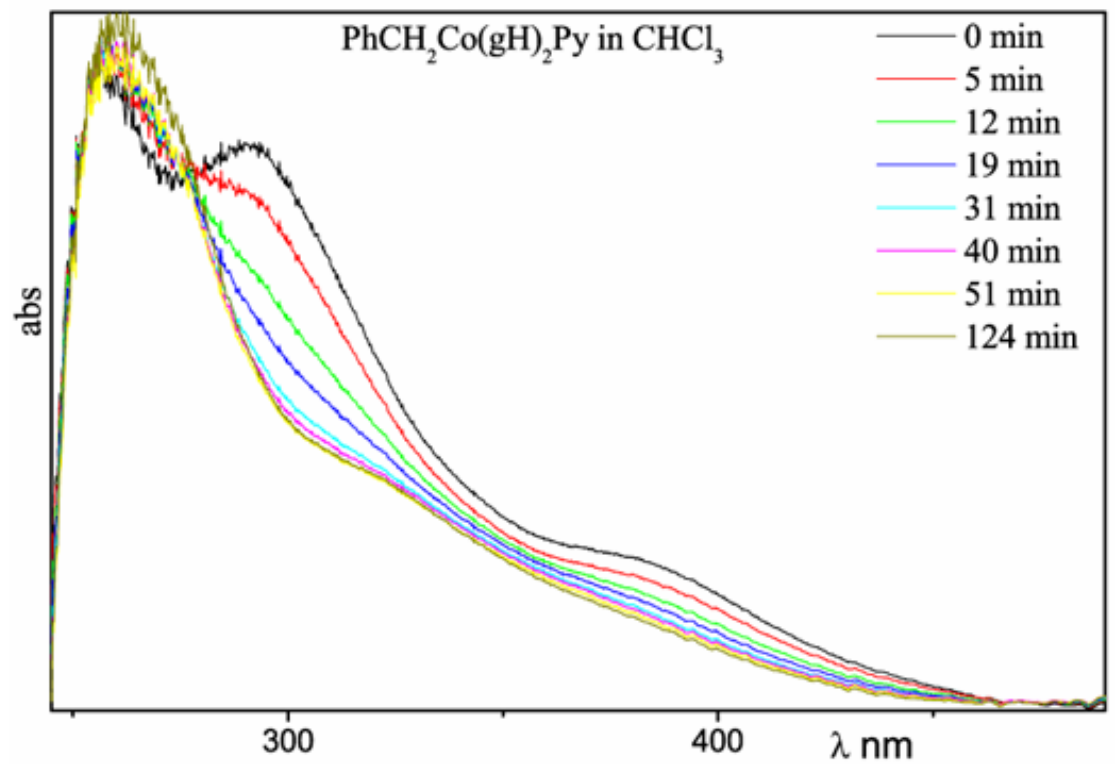

Figure S3. UV-vis spectral changes in $\mathrm{PhCH}_{2} \mathrm{Co}(\mathrm{gH})_{2} \mathrm{Py}(6)$ due to $\mathrm{O}_{2}$ insertion at different time interval (in $\mathrm{CHCl}_{3}$ )

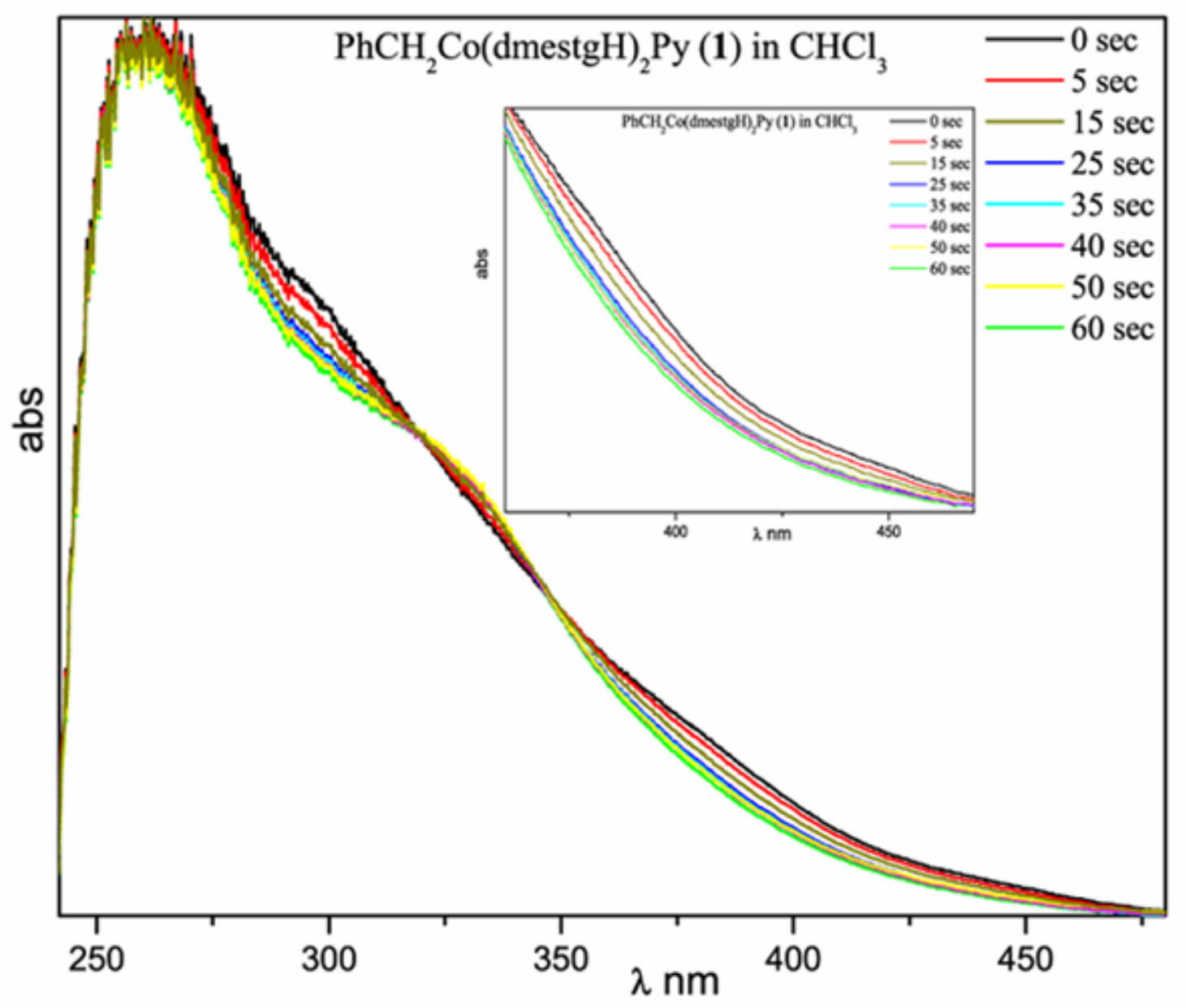

Figure S4. UV-vis spectral changes in $\mathrm{PhCH}_{2} \mathrm{Co}(\mathrm{dmestgH})_{2} \mathrm{Py}(\mathbf{1})$ due to $\mathrm{O}_{2}$ insertion at different time interval (in $\mathrm{CHCl}_{3}$ ) (Inset $350-480 \mathrm{~nm}$ ) enlargement) 


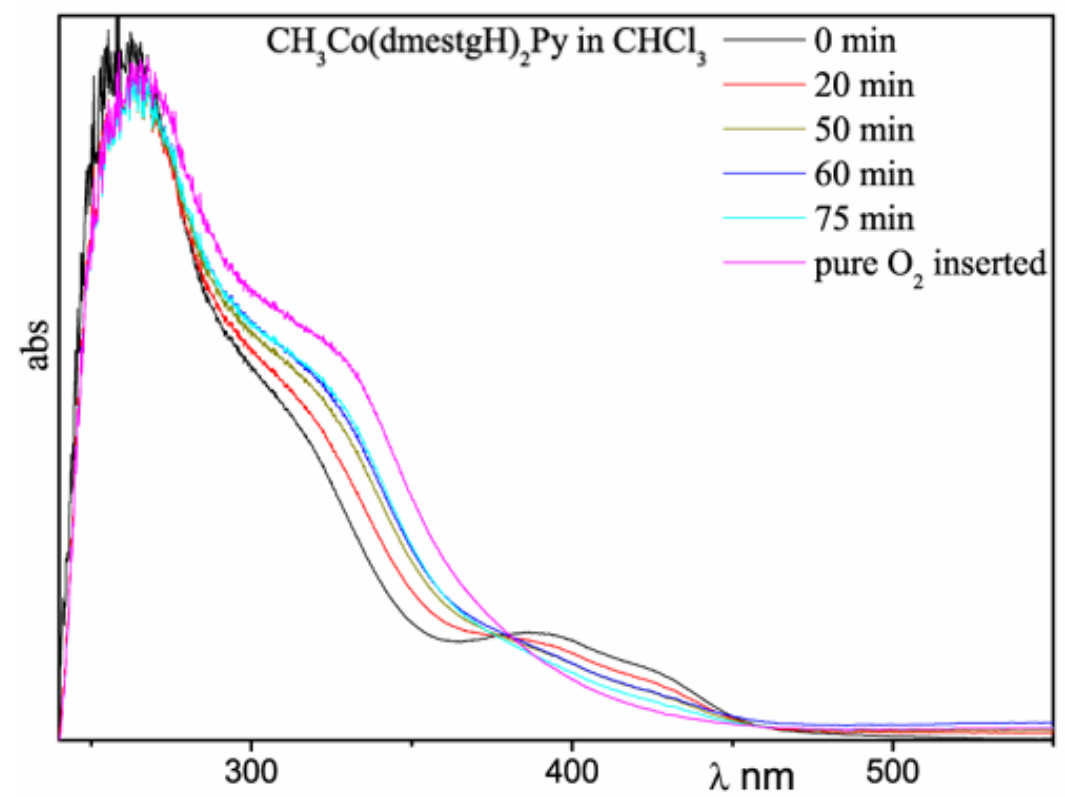

Figure S5. Uu-vis spectral changes in $\mathrm{CH}_{3} \mathrm{Co}(\mathrm{dmestgH})_{2} \mathrm{Py}$ due to $\mathrm{O}_{2}$ insertion at different time interval (in $\mathrm{CHCl}_{3}$ )

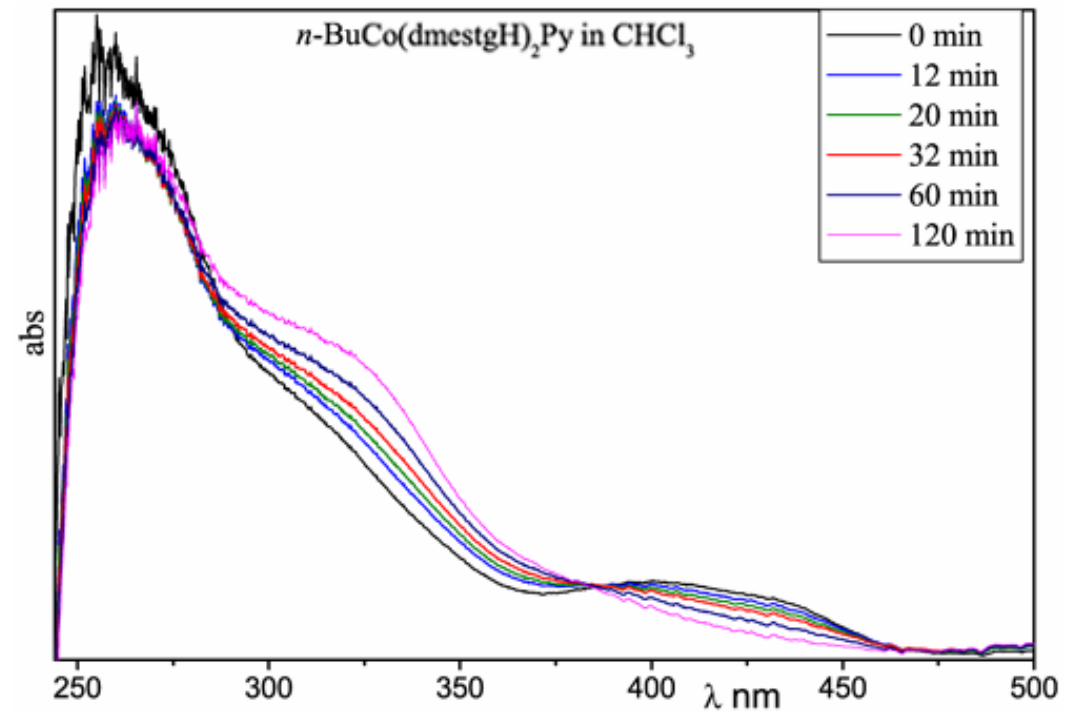

Figure S6. UV-vis spectral changes in $n$ - $\mathrm{BuCo}(\mathrm{dmestg} \mathrm{H})_{2} \mathrm{Py}$ due to $\mathrm{O}_{2}$ insertion at different time interval (in $\mathrm{CHCl}_{3}$ ) 


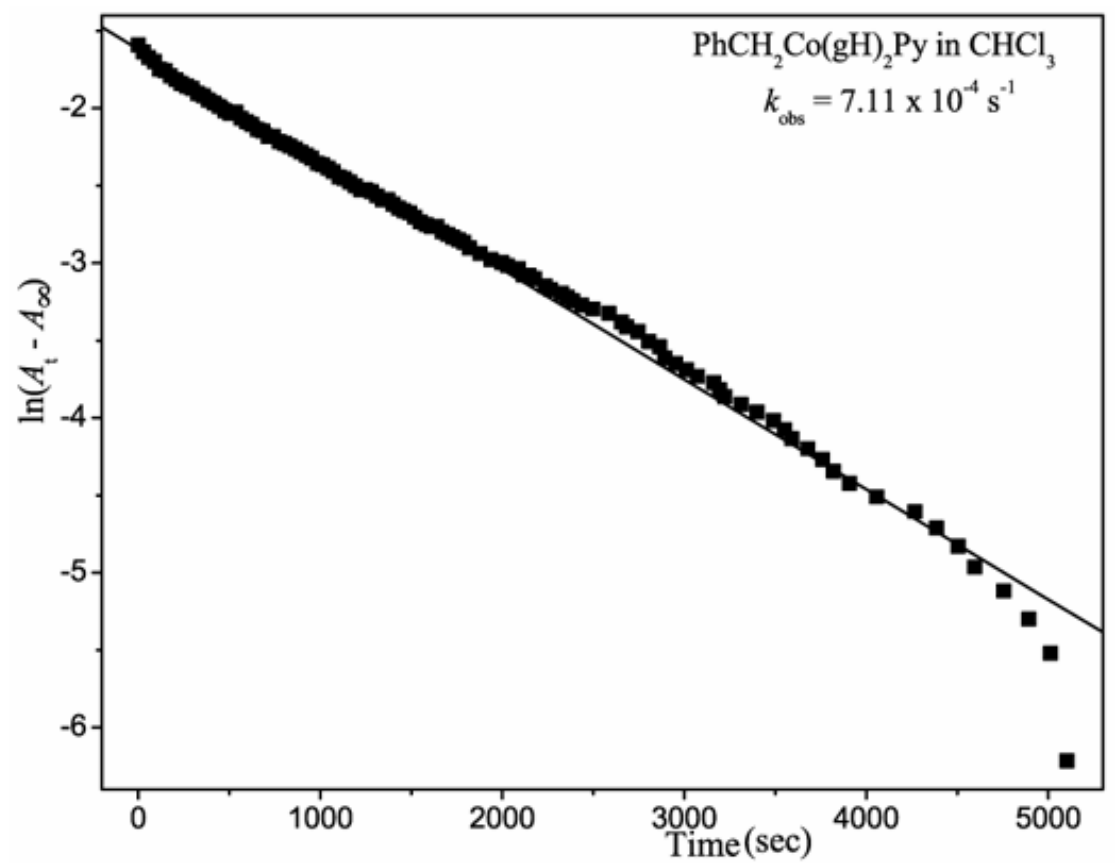

Figure S7. Plot of $\ln \left(A_{\mathrm{t}}-A_{\infty}\right)$ vs. time(sec) for $\mathrm{PhCH}_{2} \mathrm{Co}(\mathrm{gH})_{2} \mathrm{Py}(6)$ (in $\mathrm{CHCl}_{3}$ )

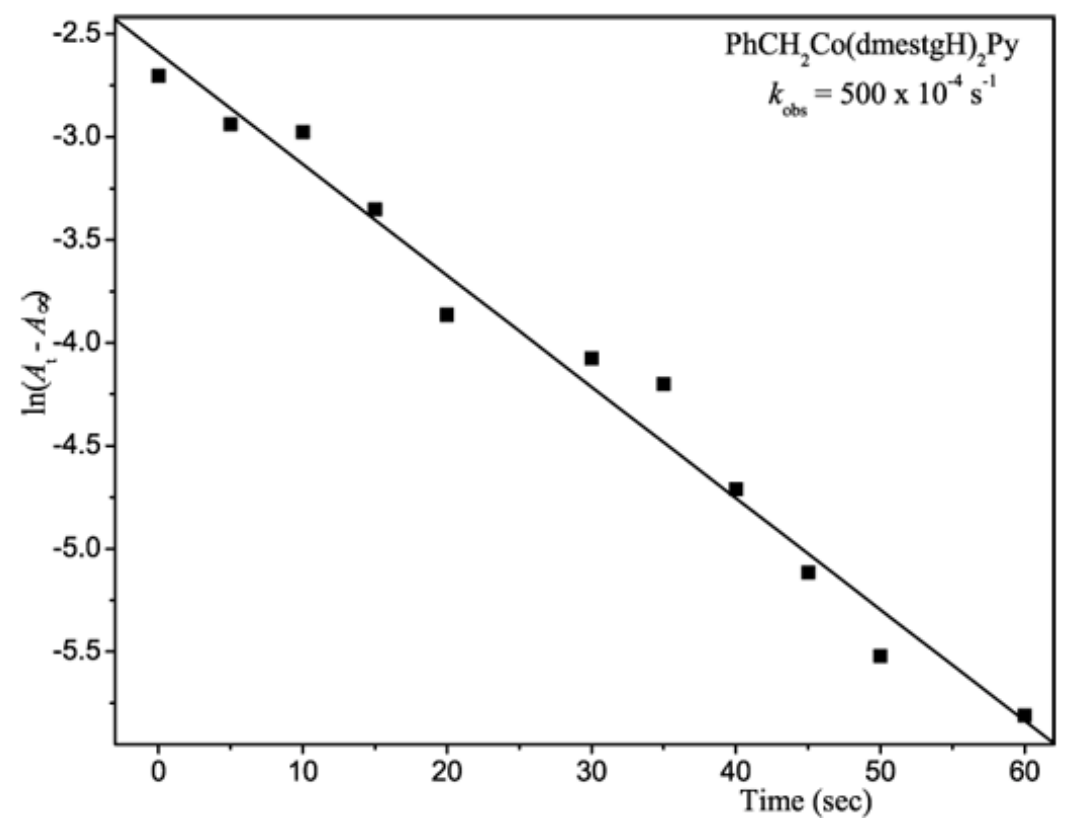

Figure S8. Plot of $\ln \left(A_{\mathrm{t}}-A_{\infty}\right)$ vs. time(sec) for $\mathrm{PhCH}_{2} \mathrm{Co}(\operatorname{dmestgH})_{2} \mathrm{Py}(\mathbf{1})$ (in $\mathrm{CHCl}_{3}$ ) 


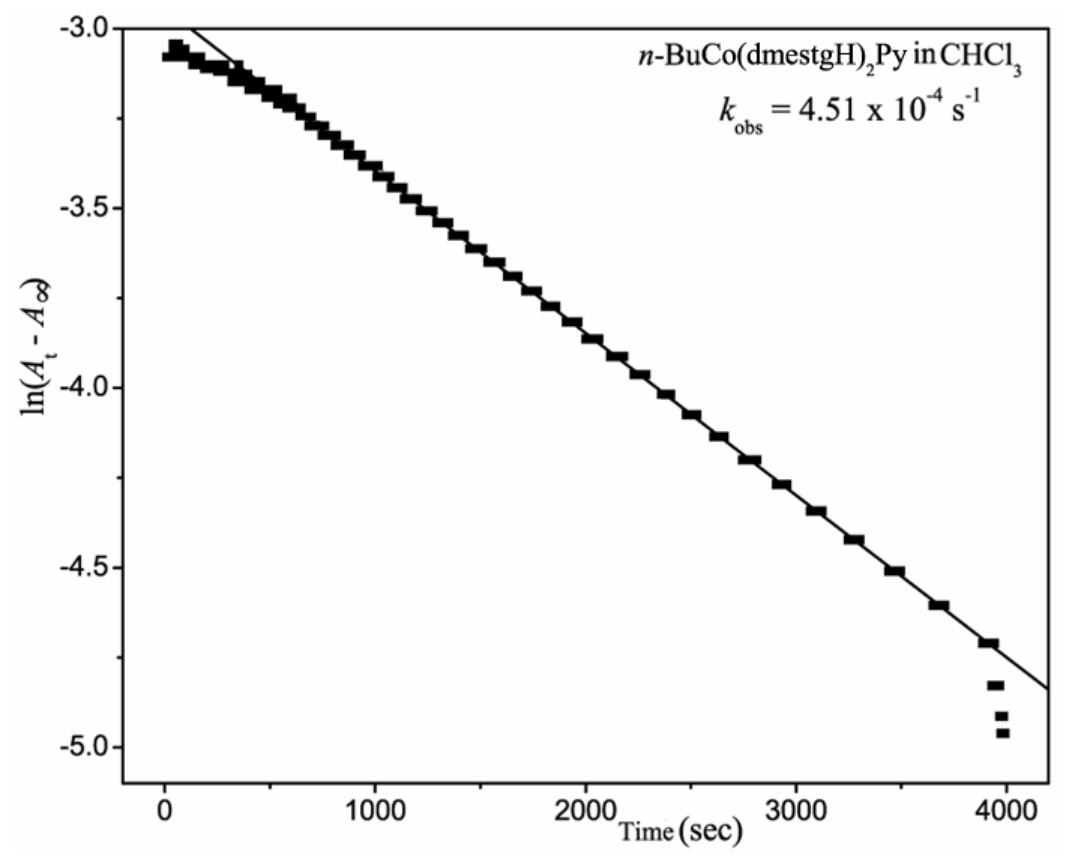

Figure S9. Plot of $\ln \left(A_{t}-A_{\infty}\right)$ vs. time(sec) for $n-\mathrm{BuCo}(\operatorname{dmestgH})_{2} \mathrm{Py}\left(\mathrm{in} \mathrm{CHCl}_{3}\right)$

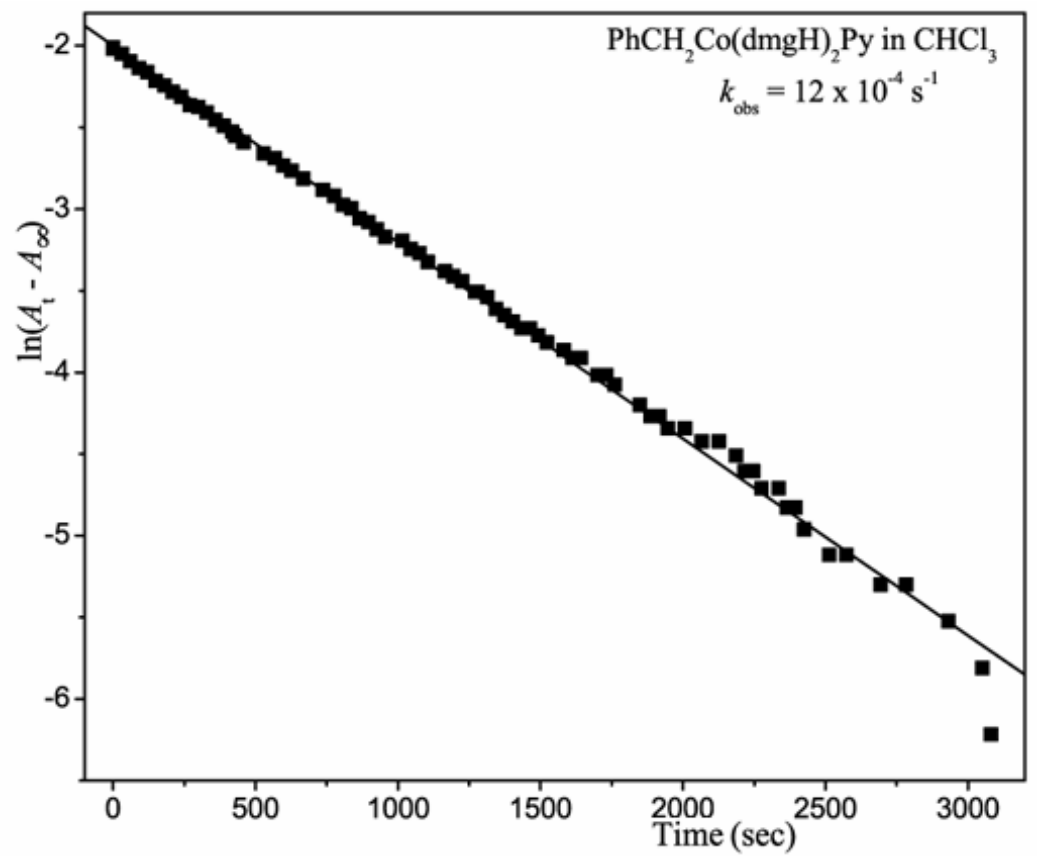

Figure S10. Plot of $\ln \left(A_{\mathrm{t}}-A_{\infty}\right)$ vs. time( $\left.\mathrm{sec}\right)$ for $\mathrm{PhCH}_{2} \mathrm{Co}(\mathrm{dmgH})_{2} \mathrm{Py}$ (in $\left.\mathrm{CHCl}_{3}\right)$ 


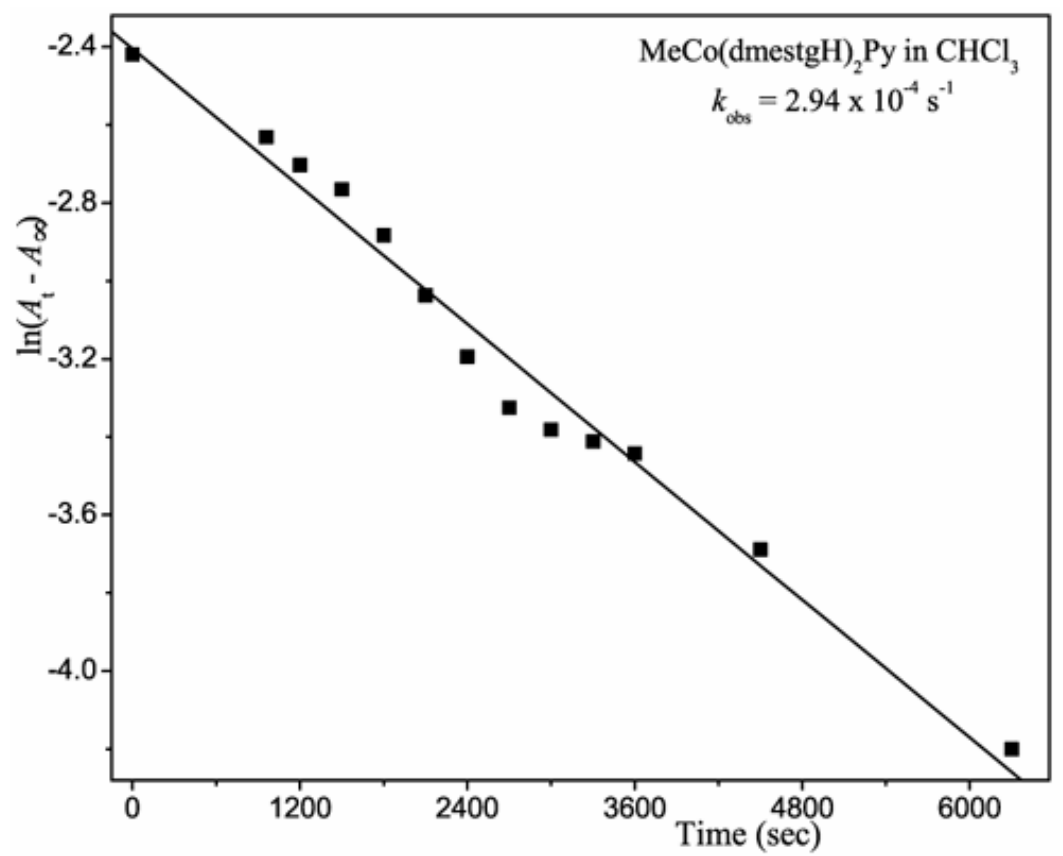

Figure S11. Plot of $\ln \left(A_{\mathrm{t}}-A_{\infty}\right)$ versus time(sec) for $\mathrm{MeCo}(\operatorname{dmestgH})_{2} \mathrm{Py}\left(\right.$ in $\left.\mathrm{CHCl}_{3}\right)$ 